\title{
The incidence of HLA-DQ2/DQ8 in Turkish children with celiac disease and a comparison of the geographical distribution of HLA-DQ
}

\author{
Ahmet Basturk, Reha Artan, Aygen Yilmaz \\ Department of Paediatric Gastroenterology, Faculty of Medicine, Akdeniz University, Antalya, Turkey
}

Gastroenterology Rev 2017; 12 (4): 256-261

DOI: https://doi.org/10.5114/pg.2017.72099

Key words: celiac disease, HLA DQ, child.

Address for correspondence: Ahmet Basturk MD, PhD, Department of Paediatric Gastroenterology, Faculty of Medicine, Akdeniz University, 07059 Antalya, Turkey, fax: +90 2422274320, e-mail: drahmetbasturk@hotmail.com

\begin{abstract}
Introduction: Celiac disease (CD) is an auto-immune enteropathy that occurs in genetically pre-disposed people as a result of the consumption of gluten-containing foods.

Aim: To identify the incidence of HLA-DQ2 and HLA-DQ8 observed in children with CD.

Material and methods: In this study, we focused on children ranging in age from 2 to 18 years and diagnosed with celiac disease. In our patients diagnosed with CD, in addition to tissue transglutaminase antibodies (anti-tTG), we also evaluated HLADQ2 B1 and HLA-DQ8 B1 alleles using the method of polymerase chain reaction (PCR)/sequence-specific oligonucleotide probes (Luminex ${ }^{\circledast}$ ). The detection of 0201/0202 for HLA-DQ2 allele and 0302 for HLA-DQ8 allele was accepted as a positive result.

Results: The mean age of our patients with celiac disease was $7.42 \pm 3.18$ years, and the female/male ratio was $1.5 / 1$. Seventy-six percent of our patients were HLA-DQ2 and/or HLA-DQ8 positive, 67\% were HLA-DQ2 positive, and 25\% were HLA-DQ8 positive. Nevertheless, $24 \%$ of them were HLA-DQ2 and HLA-DQ8 negative. The incidence of HLA-DQ2 in the control group was $18.8 \%$ with a significant difference compared to the HLA-DQ2 incidence in the patient group $(67 \%)(p<0.05)$. Similarly the HLA-DQ8 incidence in the control group (5.7\%) was significantly lower than the incidence in the patient group $(25 \%)(p<0.05)$.

Conclusions: The incidence of the patients diagnosed with CD, who are HLA-DQ2 and HLA-DQ8 negative, varies among different populations.
\end{abstract}

\section{Introduction}

Celiac disease (CD) is an auto-immune enteropathy that occurs in genetically pre-disposed people as a result of the consumption of gluten-containing foods. Gluten is a protein found in wheat and in other grains (e.g. barley, rye, oats). Celiac disease is observed in both children and adults and is a lifelong disorder [1].

Major histocompatibility complex (MHC) is a gene found in all vertebrates, and it has certain functions related to the immunity in some of them. The human version of $\mathrm{MHC}$ is $\mathrm{HLA}$ [2]. In humans, the gene region encoding $\mathrm{MHC}$ antigens is a sequential DNA locus close to the centromere on the short arm of chromosome 6 [3]. HLA antigens are categorised into three groups as class I, class II, and class III according to their structures and functions [4]. Regarding the celiac disease, the basic determinants of the genetic susceptibility for celiac disease are the MHC class II HLA-DQA and DQB genes (these genes are encoded by the histocompatibility region on the short arm of chromosome 6) [5].

The role of tissue groups in the diagnosis of celiac disease gains increasing importance. HLA-DQ2 and/ or HLA-DQ8 have low specificity for CD (54\% on average), and their value for the prediction of celiac disease is low. On the other hand, studies have shown that the susceptibility of HLA-DQ2 and DQ8 in CD is high (96.2\% on average) and the possibility of celiac disease in people who are negative for both of these alleles is extremely low [6, 7].

The golden standard in the diagnosis of celiac disease is small bowel biopsy. Currently, small bowel biopsy is recommended for cases with positive serology or with negative serology but strong clinical suspicion [8]. Typical findings in celiac disease are increased intraepi- 
thelial lymphocytes, crypt hyperplasia, and total villous atrophy (also called "flat mucosa") [9].

Presently there is no consensus on diagnosing CD without carrying out small bowel biopsy. The last version of ESPGHAN's guideline states that patients with apparent clinical symptoms and with highly positive titration of anti-tTG IgA (10x higher than normal) with positive endomysial antibodies (EMA) and with positive HLA-DQ2 and/or HLA-DQ8 heterodimer can be diagnosed without a biopsy after negotiation with the patient's family. However, in patients with low titration of anti-tTG antibodies and negative EMA test, biopsy is definitely necessary. In patients diagnosed without biopsy, significant relief of symptoms and normalisation in $\mathrm{CD}$ antibodies should be closely monitored [8].

Regarding these data, analysis of HLA-DQ2 and HLA-DQ8 haplotypes in celiac disease is essential for the diagnosis and prevention of misdiagnosis. Although there are some studies focused on this subject in the literature, further studies are needed.

\section{Aim}

The aim of our study was to identify the incidence of HLA-DQ2 and HLA-DQ8 tissue groups in the patients diagnosed with $C D$.

\section{Material and methods}

In this study, we focused on children ranging in age from 2 to 18 years, diagnosed with celiac disease in the Akdeniz University Paediatric Gastroenterology Outpatient Clinic between September 2010 and May 2015. We obtained the approval of the Local Ethics Committee for the clinical trials of Akdeniz University before the study startup (Date: June 10, 2015, Approval Number: 278). In the Paediatric Gastroenterology Outpatient Clinic of our hospital, for diagnostic purposes, we evaluated antitTG IgA, anti-tTG IgG antibodies, and HLA-DQ2 and HLA-DQ8 alleles with the "polymerase chain reaction


od $\left(\right.$ Luminex $\left.^{\circledR}\right)$ in patients with a suspicion of CD. For a definitive diagnosis, the histopathological analysis of multiple duodenal biopsies was done. Regarding the results of the histopathological analysis, intraepithelial lymphocytosis is classified as Marsh 1, a moderate decrease of the ratio of villus length/crypt depth as Marsh 2, partial villous atrophy as Marsh 3a, subtotal villous atrophy as Marsh $3 \mathrm{~b}$, and total villous atrophy as Marsh 3c [8]. In patients diagnosed with celiac disease, the obtained HLA-DQ2 and HLA-DQ8 allele results are retrospectively evaluated and negative and positive results are determined. During the interpretation of the HLA-DQ2 and HLA-DQ8 allele levels, the detection of 0201 and 0202 for HLA-DQ2 B1 and the detection of
0302 for HLA-DQ8 B1 are regarded as positive [10]. As a control group for HLA-DQ2, we evaluated the data of a postgraduate thesis focused on the HLA antigen levels of 771 subjects in the Immunology Department of the Central Laboratory in the Balcalı Hospital of the Medical Faculty of the Çukurova University [11]. As a control group for HLA-DQ8, we evaluated the data of a similar postgraduate thesis focused on the HLA antigen levels of 910 subjects in the Immunology Department of the Central Laboratory in the Medical Faculty of the Çukurova University [12]. We compared the HLA-DQ2 B1 and HLA-DQ8 B1 results of our patients diagnosed with CD with the HLA-DQ2 [11] and HLA-DQ8 [12] results of the healthy populations and assessed the incidence and significance of the HLA-DQ2 B1 and HLA-DQ8 B1 alleles in our patients diagnosed with celiac disease.

\section{Statistical analysis}

We used the software SPPS ver. 15.0 (SPSS Inc., Chicago, IL, USA). The comparative analysis of the data was performed with $\chi^{2}$ test and Fisher's Exact test. For the descriptive statistical analysis of the data, we used average \pm standard deviation for the numeric variables and numbers and percentages for the categorical variables. The accepted significance level was $p<0.05$.

\section{Results}

One hundred and twenty-nine patients diagnosed with celiac disease were enrolled into our study. Twenty-seven of these patients were not included in the evaluation because their HLA-DQ2 B1 and HLA-DQ8 B1 alleles were not investigated, but they had positive anti-tTG antibodies and they were diagnosed with CD. The remaining 102 patients were included in the study, as their diagnosis was confirmed with the clinical, laboratory, and histopathological findings. The mean age of our patients with celiac disease was $7.42 \pm 3.18$ years and the female/male ratio was $1.5 / 1$. In our study, the most frequently observed complaints were diarrhoea (83\%), abdominal pain (40\%), growth retardation (38\%), and abdominal distension (41\%). Analysis of the histopathological classification showed that $68.9 \%$ of our patients had Marsh 3, 2.4\% had Marsh 2, and 9.7\% had Marsh 1. The analysis of our patients with CD according to the tissue groups revealed that $76 \%$ of them were HLA-DQ2 and HLA-DQ8 positive, $67 \%$ of them were HLA-DQ2 positive, and $25 \%$ were HLA-DQ8 positive. However, $24 \%$ of our patients were neither HLA-DQ2 nor HLA-DQ8 positive. The comparative analysis with the control group [11] showed that the incidence of HLA-DQ2 in the control group was $18.8 \%$, but it was $67 \%$ in the patient group. The difference between these groups was signifi- 
cant $(p<0.05)$. A similar significance was also observed for the incidence of HLA-DQ8 between the control group [12] (57\%) and the patient group (25\%) ( $p<0.05)$. The complaints during the outpatient visits, average ages, and distribution according to the histopathological classification of patients with HLA-DQ2, HLA-DQ8,

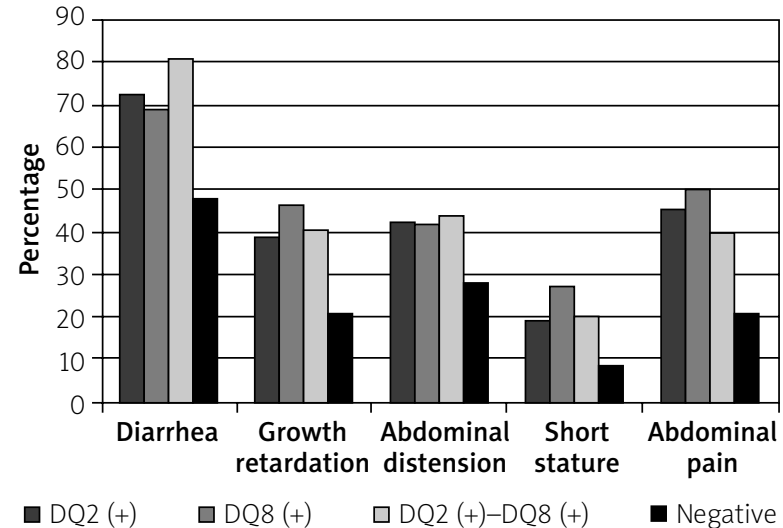

Figure 1. The distribution of celiac groups regarding the complaints of the patients during their outpatient visits
HLA-DQ2, and/or HLA-DQ8 and without HLA-DQ2 and HLA-DQ8 and their comparison is listed in Table I.

The comparative evaluation of our patients with CD regarding the HLA-DQ2, HLA-DQ8, HLA-DQ2, and/ or HLA-DQ8 did not show any significant difference. On the other hand, we observed that complaints of the patients without HLA-DQ2 and HLA-DQ8 were less frequent (Figure 1).

\section{Discussion}

There are numerous studies in the literature focused on the incidence of HLA-DQ in patients diagnosed with celiac disease. In the majority of these studies HLA-DQ was evaluated, and in some studies only HLA-DQ A1 or B1 alleles were evaluated.

One of the HLA-DQ studies was conducted by Karell et al. [6] In this study, which was done in France, Italy, Finland, Norway, and England, the incidences were between $87 \%$ and $93.7 \%$ for HLA-DQ2, between $5 \%$ and $8 \%$ for HLA-DQ8, and between $89.4 \%$ and $96.7 \%$ for HLA-DQ2 and/or HLA-DQ8. In the study of Vidales et al. [13], the determined incidences were $93.4 \%$ for HLA-DQ2; $2.4 \%$ for HLA-DQ8, and 95.6\% for HLA-DQ2 and/or HLA-DQ8. The incidences of another study by

Table I. The distribution of the celiac patients and groups regarding the demographic characteristics, complaints during the visits, and histopathological classifications

\begin{tabular}{|c|c|c|c|c|c|}
\hline Parameter & DQ2 (+) & DQ8 (+) & $\mathrm{DQ} 2$ and/or DQ8 (+) & DQ2 and DQ8 (-) & Total \\
\hline Age, average & $6.97 \pm 3.32$ & $7.12 \pm 2.78$ & $7.08 \pm 2.94$ & $6.85 \pm 3.24$ & $7.42 \pm 3.18$ \\
\hline Gender (M/F) & $43 / 25$ & $15 / 11$ & $44 / 31$ & $15 / 10$ & $65 / 37$ \\
\hline \multicolumn{6}{|l|}{ Complaints during visits (\%): } \\
\hline Diarrhoea & 73 & 69 & 81 & 48 & 83 \\
\hline Growth retardation & 40 & 46 & 40 & 20 & 38 \\
\hline Abdominal distension & 43 & 42 & 43 & 28 & 41 \\
\hline Short stature & 19 & 27 & 19 & 8 & 18 \\
\hline Chronic abdominal pain & 46 & 50 & 40 & 20 & 40 \\
\hline Delayed puberty & 1 & 4 & 1 & 0 & 1 \\
\hline Iron deficiency anaemia & 2 & 4 & 2 & 0 & 2 \\
\hline Constipation & 0 & 0 & 0 & 0 & 0 \\
\hline Hypertransaminasaemia & 2 & 0 & 0 & 0 & 1 \\
\hline Recurrent oral aphthae & 0 & 0 & 0 & 0 & 0 \\
\hline \multicolumn{6}{|c|}{ Histopathological classification (\%): } \\
\hline Marsh 1 & 5 & 9 & 8 & 11 & 9.7 \\
\hline Marsh 2 & 21 & 18 & 23 & 21 & 21.4 \\
\hline Marsh 3 & 74 & 62 & 65 & 66 & 68.9 \\
\hline
\end{tabular}


Table II. Studies related with HLA-DQ2 and/or HLA-DQ8 in patients with celiac disease

\begin{tabular}{|c|c|c|c|c|c|}
\hline $\begin{array}{l}\text { Author, year } \\
\text { (reference no.) }\end{array}$ & Country & $\begin{array}{l}\text { Patient (P)/ } \\
\text { Control (C) }\end{array}$ & $\begin{array}{l}\text { HLA-DQ2 (+) } \\
\text { P/C (\%) }\end{array}$ & $\begin{array}{c}\text { HLA-DQ8 (+) } \\
\text { P/C (\%) }\end{array}$ & $\begin{array}{c}\text { HLA-DQ2 and/or } \\
\text { DQ8 (+) } \\
\text { P/C (\%) }\end{array}$ \\
\hline Djilali-Saiah, 1998 [19] & France & $\begin{array}{c}101 / 130 \\
D Q B 1 \\
D Q A 1 B 1\end{array}$ & $\begin{array}{c}68 / 43 \\
70.2 / 34.6\end{array}$ & & \\
\hline Pérez-Bravo, 1999 [27] & Chile & $\begin{array}{c}62 / 124 \\
D Q A 1 B 1 \\
\end{array}$ & $11.3 /$ & $25.8 /$ & $37.1 /$ \\
\hline Sumnik, 2000 [28] & Czechoslovakia & $\begin{array}{c}15 / 186 \\
D Q A 1 B 1 \\
\end{array}$ & $80 / 49$ & $67 / 66$ & $100 /$ \\
\hline Tümer, 2000 [18] & Turkey & $\begin{array}{c}33 / 77 \\
D Q A 1 B 1\end{array}$ & $52 / 34$ & & \\
\hline Neuhausen, 2002 [29] & Israel & $\begin{array}{c}23 / \\
D Q A 1 B 1\end{array}$ & $82.6 /$ & $56.5 /$ & $100 /$ \\
\hline Kaur, 2002 [20] & India & $\begin{array}{c}35 / 135 \\
D Q A 1 B 1 \\
D Q B 1\end{array}$ & $\begin{array}{l}97.1 / \\
100 /\end{array}$ & & \\
\hline \multirow[t]{5}{*}{ Karell, 2003 [6] } & France & $\begin{array}{c}92 / \\
D Q A 1 B 1 \\
\end{array}$ & $87 /$ & $6.5 /$ & $93.5 /$ \\
\hline & Italy & $\begin{array}{c}302 / \\
D Q A 1 B 1\end{array}$ & $93.7 /$ & $5.6 /$ & $89.4 /$ \\
\hline & Finland & $\begin{array}{c}100 / \\
D Q A 1 B 1\end{array}$ & $91 /$ & $5 /$ & $96 /$ \\
\hline & Norway & $\begin{array}{c}326 / \\
D Q A 1 B 1\end{array}$ & $91.4 /$ & 5.21 & $96.6 /$ \\
\hline & England & $\begin{array}{c}188 / \\
D Q A 1 B 1\end{array}$ & $87.8 /$ & $8 /$ & $96.7 /$ \\
\hline Johnson, 2004 [30] & New York/Paris & $\begin{array}{c}44 / 66 \\
D Q A 1 B 1\end{array}$ & $86 / 93$ & $41 / 21$ & \\
\hline Vidales, 2004 [13] & Spain & $\begin{array}{c}136 / \\
D Q A 1 B 1\end{array}$ & $93.4 /$ & $2.1 /$ & $95.6 /$ \\
\hline Kuloğlu, 2008 [17] & Turkey & $\begin{array}{l}75 / 100 \\
D Q A 1 B 1\end{array}$ & $84.7 / 27$ & & \\
\hline El-Akawi, 2010 [31] & Jordan & $\begin{array}{c}44 / 53 \\
D Q A 1 B 1 \\
\end{array}$ & $80 / 32$ & & \\
\hline Piccini, 2012 [14] & Italy & $\begin{array}{c}89 / 70 \\
D Q A 1 B 1\end{array}$ & $64 / 21.3$ & $16.8 / 10$ & $77.5 / 30$ \\
\hline Erriu, 2013 [21] & Italy & $\begin{array}{c}44 / \\
D Q B 1\end{array}$ & $79.5 /$ & & \\
\hline Rostami-Nejad, 2014 [26] & Iran & $\begin{array}{c}59 / 151 \\
D Q A 1 B 1 \\
\end{array}$ & $83.03 / 35.09$ & $25.4 /$ & $97 / 58$ \\
\hline Pallav, 2014 [15] & USA & $\begin{array}{c}72 / 44 \\
D Q A 1 B 1\end{array}$ & $69.5 /$ & $25.3 /$ & $95.5 / 30$ \\
\hline Delgado, 2014 [16] & Spain & $\begin{array}{c}91 / 158 \\
D Q A 1 B 1 \\
\end{array}$ & $96.7 / 63.3$ & $4.4 / 15.2$ & $100 / 74$ \\
\hline Zamani, 2014 [22] & Iran & $\begin{array}{c}120 / 100 \\
D Q B 1\end{array}$ & $75 /$ & $14 /$ & $98 / 44$ \\
\hline Our study, 2015 & Turkey & $\begin{array}{c}102 / \\
D Q 2-771 \\
D Q 8-910 \\
D Q B 1\end{array}$ & $67 / 18.8$ & $25 / 5.7$ & $76 /$ \\
\hline
\end{tabular}


Piccini et al. [14] were 64\% for HLA-DQ2, 16.8\% for HLADQ8, and 77.5\% for HLA-DQ2 and/or HLA-DQ8.

Pallav et al. [15] obtained similar results regarding the incidences: $69.5 \%$ for HLA-DQ2, 25.3\% for HLA-DQ8, and $95.5 \%$ for HLA-DQ2 and/or HLA-DQ8.

Concurrently in a study, Delgado et al. [16] found the following incidences: $96.7 \%$ for HLA-DQ2, 4.4\% for HLADQ8, and $100 \%$ for HLA-DQ2 and/or HLA-DQ8.

In some of the studies focused on patients with celiac disease and conducted in our country, Kuloğlu et al. [17] reported the incidence of HLA-DQ2 as 84.7\%; Tümer et al. [18] reported the incidence of HLA-DQ2 as 52\%.

Among the studies on HLA-DQ and HLA-DQ B1, Djilali-Saiah et al. [19] found that $70.2 \%$ of the patients with CD had HLA-DQ2, and 68\% had HLA-DQ2 B1. In another study Kaur et al. [20] showed that $97.1 \%$ of the patients diagnosed with CD had HLA-DQ2 and $100 \%$ of them had HLA-DQ2 B1.

Among the studies evaluating only HLA-DQ B1, Erriu et al. [21] reported that $79.5 \%$ of the patients diagnosed with CD and Zamani et al. [22] reported that $75 \%$ of the patients diagnosed with CD had HLA-DQ2, 14\% had HLA-DQ8, and 98\% had HLA-DQ2 and/or HLA-DQ8.

The comparison of all these studies reveals that the results of the studies evaluating HLA-DQ haplotype and HLA-DQ B1 allele are similar. In our study, the results regarding the incidence of HLA-DQ2 was $67 \%$ and the incidence of HLA-DQ2 and/or HLA-DQ8 was $76 \%$, and they were in line with the results reported in the literature.

Surprisingly, the incidence of HLA-DQ8 in our study (25\%) was much higher than the studies in the literature reporting lower incidences (Cameroon 0.6\%; Italy $2 \%$; Hungary $2.3 \%$; USA $4.2 \%$; Finland $6.4 \%$; Japan $7.6 \%)[23,24]$. However, our result is in line with the result of the study conducted on HLA-DQ8 by Catassi et al. [25] in 2009 on Turkish people (22\%), North American natives (25.3\%), Mexicans (28.3\%), and Bushmen (an indigenous group of southern Africa) (30\%). In addition to that, our results also confirm the results of Rostami-Nejad et al. [26] on Iranian people (25.4\%) and the results of Pérez-Brave et al. [27] in Chile (25.8\%).

There are some published studies in the literature reporting higher incidences of HLA-DQ8 than the incidences of our study. In the study conducted by Sumnik et al. [28] in Czechoslovakia in 2000, the incidence of HLA-DQ8 was $67 \%$ and in the study conducted by Neuhausen et al. [29] in Israel with Bedevins the incidence of HLA-DQ8 was $56.5 \%$.

All these results confirm that the incidence of HLADQ8 varies in different geographical regions.

According to the data originating from western countries $[4,13,15,16,28]$, the percentage of patients with celiac disease that are HLA-DQ2 and HLA-DQ8 negative is between 0 and $10 \%$, but there are also other studies reporting higher percentages. In their study conducted in Chile, Pérez-Bravo et al. [27] reported that $62.9 \%$ of patients were HLA-DQ2 and HLA-DQ8 negative. The result reported by Piccini et al. [14] and obtained in Italy (22.5\%) is in line with our result (24\%). These results show that the incidence of patients diagnosed with celiac disease and who are HLA-DQ2 and HLA-DQ8 negative varies among different populations.

The HLA DQ results of the patients with celiac disease in our study and in other studies are shown in Table II.

\section{Conclusions}

The incidence of the patients diagnosed with $C D$, who are HLA-DQ2 and HLA-DQ8 negative, varies among different populations. Therefore, it is believed that inherited celiac disease, besides HLA-DQ, may also be closely related to other tissue groups and non-HLA genes with an increasing importance recognised in recent years.

\section{Conflict of interest}

The authors declare no conflict of interest.

\section{References}

1. Trancone R, Jabri B. Celiac disease and gluten sensitivity. J Intern Med 2011; 269: 582-90.

2. Khan AN, Gregorie CJ, Tomasi TB. Histone deacetylase inhibitors induce TAP, LMP, Tapasin genes and MHC class I antigen presentation by melanoma cells. Cancer Immunol Immunother 2008; 57: 647-54.

3. Complete sequence and gene map of a human major histocompatibility complex. The MHC sequencing consortium. Nature 1999; 401: 921-3.

4. Sanfilipo F, Vaughn WK, Spees EK, et al. Benefits of HLA A and HLA B matching of graft and patient out come after cadaveric donor renal transplantation. N Eng J Med 1984; 311: 358-64.

5. Mearin ML, Biemond I, Peña AS, et al. HLA-DR phenotypes in Spanish celiac children: their contribution to the understanding of the genetics of the disease. Gut 1983; 24: 532-7.

6. Karell K, Louka AS, Moodie SJ, et al. HLA types in celiac disease patients not carrying the DQA1_05-DQB1_02 (DQ2) heterodimer: results from the European Genetics Cluster on Celiac Disease. Hum Immunol 2003; 64: 469-77.

7. Book L, Hart A, Black J, et al. Prevalance and clinical characteristics of celiac disease in Down syndrome in a US study. Am J Med Genet 2001; 98: 70-4.

8. Husby S, Koletzko S, Korponay-Szabó IR, et al. ESPGHAN Working Group on Celiac Disease Diagnosis; ESPGHAN Gastroenterology Committee. European Society for Pediatric Gastroenterology, Hepatology, and Nutrition guidelines for the diagnosis of celiac disease. J Pediatr Gastroenterol Nutr 2012; 54: 136-60. 
9. Ensari A. Gluten sensitive enteropathy: controversies in diagnosis and classification. Arch Pathol Lab Med 2010; 134: 826-36.

10. Klitz W, Maiers M, Spellman S, et al. New HLA haplotype frequency reference standards: high-resolution and large sample typing of HLA DR-DQ haplotypes in a sample of European Americans. Tissue Antigens 2003; 62: 296-307.

11. Etiz P. The distribution of HLA antigens in our region. Master's thesis. Adana 2009.

12. Temiz N. Determination of types and frequencies HLA (human leukocyte antigens) in Mediterranean region. Master's thesis. Kahramanmaraş 2005.

13. Vidales MC, Zubillaga P, Zubillaga I, Alfonso-Sánchez MA. Allele and haplotype frequencies for HLA class II (DQA1 and DQB1) loci in patients with celiac disease from Spain. Hum Immunol 2004; 65: 352-8.

14. Piccini B, Vascotto $M$, Serracca L, et al. HLA-DQ typing in the diagnostic algorithm of celiac disease. Rev Esp Enferm Dig 2012; 104: 248-54.

15. Pallav K, Kabbani T, Tariq S, et al. Clinical utility of celiac dis ease-associated HLA testing. Dig Dis Sci 2014; 59: 2199-206.

16. Delgado JF, Amengual MJ, Veraguas A, et al. Paediatric celiac patients carrying the HLA-DR7-DQ2 and HLA-DR3-DQ2 haplotypes display small clinical differences. Acta Paediatr 2014; 103: e238-42.

17. Kuloğlu Z, Doğanci T, Kansu A, et al. HLA types in Turkish children with celiac disease. Turk J Pediatr 2008; 50: 515-20.

18. Tümer L, Altuntaş B, Hasanoglu A, et al. Pattern of human leukocyte antigens in Turkish children with celiac disease. Pediatr Int 2000; 42: 678-81.

19. Djilali-Saiah I, Schmitz J, Harfouch-Hammoud E, et al. CTLA-4 gene polymorphism is associated with predisposition to celiac disease. Gut 1998; 43: 187-9.

20. Kaur G, Sarkar N, Bhatnagar S, et al. Pediatric celiac disease in India is associated with multiple DR3-DQ2 haplotypes. Hum Immunol 2002; 63: 677-82.

21. Erriu M, Abbate GM, Pili FM, et al. Oral signs and HLA-DQB1 02 haplotypes in the celiac paediatric patient: a preliminary study. Autoimmune Dis 2013; 2013: 389590.

22. Zamani M, Modares-Sadegi M, Shirvani F, et al. The involvement of the HLA-DQB1 alleles in the risk and the severity of Iranian celiac disease patients. Int J Immunogenet 2014; 41: 312-7.

23. Koskinen L, Romanos J, Kaukinen K, et al. Cost-effective HLA typing with tagging SNPs predicts celiac disease risk haplotypes in the Finnish, Hungarian, and Italian populations. Immunogenetics 2009; 61: 247-56.

24. Alarida K, Harown J, Di Pierro MR, et al. HLA-DQ2 and -DQ8 genotypes in celiac and healthy Libyan children. Dig Liver Dis 2010; 42: 425-7.

25. Catassi C, Yachha SK. The global village of celiac disease. In: Frontiers in Celiac Disease. Fasano A, Troncone R, Branski D (eds). Karger Basel, Switzerland 2008; 23-31.

26. Rostami-Nejad M, Romanos J, Rostami K, et al. Allele and haplotype frequencies for HLA-DQ in Iranian celiac disease patients. World J Gastroenterol 2014; 20: 6302-8.

27. Pérez-Bravo F, Araya M, Mondragón A, et al. Genetic differences in $\mathrm{HLA}-\mathrm{DQA} 1^{*}$ and $\mathrm{DQB} 1^{*}$ allelic distributions between celiac and control children in Santiago, Chile. Hum Immunol 1999; 60: 262-7.

28. Sumník Z, Kolousková S, Cinek O, et al. HLA-DQA ${ }^{\star} 05$ $\mathrm{DQB} 1^{\star} 0201$ positivity predisposes to celiac disease in Czech diabetic children. Acta Paediatr 2000; 89: 1426-30.

29. Neuhausen SL, Weizman Z, Camp NJ, et al. HLA DQA1-DQB1 genotypes in Bedouin families with celiac disease. Hum Immunol 2002; 63: 502-7.

30. Johnson TC, Diamond B, Memeo L, et al. Relationship of HLADQ8 and severity of celiac disease: comparison of New York and Parisian cohorts. Clin Gastroenterol Hepatol 2004; 2: 888-94.

31. El-Akawi ZJ, Al-Hattab DM, Migdady MA. Frequency of HLADQA $1^{\star} 0501$ and DQB $1^{\star} 0201$ alleles in patients with celiac disease, their first-degree relatives and controls in Jordan. Ann Trop Paediatr 2010; 30: 305-9.

Received: 19.07.2016

Accepted: 20.08 .2016 